Mots. Les langages du politique

$118 \mid 2018$

Discours post-attentats

\title{
L'attentat comme objet de discours : problématique et enjeux
}

Discourse on terror strikes: problems and stakes

El atentado como objeto de los discursos: problemática y lo que esta en juego

Gérôme Truc, Christian Le Bart et Émilie Née

(2) OpenEdition

Journals

Édition électronique

URL : https://journals.openedition.org/mots/23683

DOI : $10.4000 /$ mots.23683

ISSN : 1960-6001

Éditeur

ENS Éditions

Édition imprimée

Date de publication : 5 novembre 2018

Pagination : $9-18$

ISBN : 979-10-362-0082-3

ISSN : 0243-6450

Référence électronique

Gérôme Truc, Christian Le Bart et Émilie Née, «L'attentat comme objet de discours : problématique et enjeux », Mots. Les langages du politique [En ligne], 118 | 2018, mis en ligne le 05 novembre 2018, consulté le 23 avril 2022. URL : http://journals.openedition.org/mots/23683 ; DOI : https://doi.org/ $10.4000 /$ mots. 23683 


\section{L'attentat comme objet de discours : problématique et enjeux}

Rescapé de l'attentat djihadiste contre la rédaction de Charlie Hebdo, le journaliste et écrivain Philippe Lançon écrit dans Le lambeau : "Je ne supporte pas plus les discours anti-musulmans que les discours pro-musulmans. Le problème, ce ne sont pas les musulmans, ce sont les discours » (Lançon, 2018, p. 288). Mais le problème est aussi, pourrait-on ajouter, qu'il n'y a pas d'attentats sans discours - et qu'il ne peut pas y en avoir. Un attentat qui ne fait pas parler de lui, qui ne suscite pas de déclarations des dirigeants politiques, de revendications des terroristes, d'analyses d'experts, de réactions émues des citoyens, n'est pas un attentat: c'est un fait divers, un homicide parmi d'autres. Ce qui distingue l'acte terroriste d'autres crimes, on le sait, est son retentissement, l'écho qu'il suscite au sein de la société frappée. «Une action violente est dénommée terroriste lorsque ses effets psychologiques sont hors de proportion avec ses résultats purement physiques ", note ainsi Raymond Aron (1962, p. 176). La disproportion tient pour une large part à la médiatisation de l'action terroriste et à son indétermination, qui déclenchent presque instantanément une vaste discussion sociale : nous avons, dans cet instant, besoin de parler, pour comprendre ce qui nous arrive - qui nous attaque, et pourquoi -, et en même temps d'exprimer ce que nous ressentons, pour nous assurer que les autres ressentent bien la même chose que nous (Rimé, 2005).

Cette discussion, où les discours s'entremêlent et se répondent, est consubstantielle de l'événement et contribue à en déterminer le sens, qui ne va pas de soi. De ce point de vue, un tournant s'est opéré avec l'apparition en Europe à la fin du xIxe siècle du terrorisme "à l'aveugle». Chercher à tuer un roi ou un dirigeant politique porte moins à interprétation que de viser la foule «innocente» (Salas, 2018). Le passage du régicide à «l'attentat», au sens où

CNRS, ISP

gerome.truc@cnrs.fr

IEP de Rennes, Arènes

lbrt35@club-internet.fr

Université Paris-Est-Créteil, Céditec

emilie.nee@u-pec.fr

Mots. Les langages du politique $\mathrm{n}^{\circ} 118$ novembre $2018 \bullet 9$ 
nous entendons ce mot aujourd'hui (Malandain, 2012) ${ }^{1}$, engage en cela un surcroît de discours et de contre-discours, des débats parfois sans fin, dont les médias constituent, depuis l'apparition du phénomène, à la fois un acteur essentiel et la caisse de résonance. Il s'agit, en particulier, de comprendre qui est ainsi visé. Lorsqu'un anarchiste fait exploser une bombe dans un théâtre, comme à Barcelone en 1893, ou dans un café, comme à Paris en 1894, s'en prend-il à monsieur Tout-le-Monde, et donc à la société en général, ou à des membres particuliers de cette société, à savoir les bourgeois (Salomé, 2010 ; Ferragu, 2019) ? L'interrogation est la même aujourd'hui lorsque des islamistes ouvrent le feu sur des terrasses de café et dans une salle de concert à Paris un vendredi soir : n'est-ce pas alors la jeunesse - et une certaine jeunesse encore : urbaine, aisée, cosmopolite - et son mode de vie, qui n'est pas celui de tous les Français, qui sont spécifiquement visés ou faut-il y voir une attaque contre l'ensemble du corps social? Et lorsque les terroristes prennent pour cibles des policiers, des militaires, des juifs ou des homosexuels (que l'on pense aux attentats de Toulouse et Montauban en 2012, à ceux de janvier 2015 en région parisienne, ou à ceux d'Orlando en juin 2016), n'a-t-on pas raison de penser que c'est «toute la société » qui est néanmoins concernée - et non seulement les représentants de l'ordre, les communautés juives et homosexuelles? Le terrorisme, en sa forme moderne, révèle ainsi que, par-delà la dimension symbolique et abstraite du lien civique, nous sommes, en pratique, plus ou moins enclins à nous identifier à nos concitoyens et à nous sentir concernés par leur sort. Les attentats, en ce sens, constituent une mise à l'épreuve de la cohésion sociale (Truc, 2016). Et c'est bien pourquoi aussi ils font tant parler.

\section{Explorer la composante discursive de l’événement terroriste}

Il existe donc une composante discursive de l'événement terroriste : c'est elle, précisément, que ce dossier de Mots. Les langages du politique entend explorer. Discours des journalistes qui commentent en direct l'événement; discours des experts, convoqués pour disserter sur ses causes et conséquences; discours des représentants politiques, qui s'attachent à en fixer le sens, à conjurer les divisions qu'il pourrait faire naître et à légitimer leur action; mais discours aussi du reste de la classe politique, des terroristes eux-mêmes, des policiers et des procureurs, des intellectuels, des citoyens... Nombreux sont les acteurs qui, dans ces circonstances, prennent la parole. Les registres et tonalités de

1. Si nous désignons communément par «attentat» aujourd'hui un acte terroriste, le mot a initialement une acception plus large, comme le rappelle l'expression «attentat à la pudeur». Ainsi le Littré définit-il un attentat comme une « entreprise criminelle, contre les lois», le terme pouvant connaître en ce sens un usage figuré - désormais quasiment perdu - qui désigne un acte qui heurte la raison, viole la morale. 
leurs discours, aussi, sont variés : narratifs, interprétatifs, critiques, compassionnels, rassembleurs, vengeurs ou haineux, etc. Des registres et tonalités qui peuvent coexister dans un même discours, être propres à certains acteurs, ou se succéder au fil du temps - car les attentats, bien entendu, continuent en règle générale de faire parler bien après qu'ils ont eu lieu, ne serait-ce qu'au travers de discours commémoratifs (Simko, 2015). Ce qu'il faudrait, en somme, c'est parvenir à établir une sorte de cartographie des multiples discours qu'un attentat suscite. Si un numéro de revue ne saurait suffire à réaliser un tel projet, c'est toutefois dans cette voie que l'on aimerait ici progresser, en offrant à la fois des études de cas et quelques vues d'ensemble ${ }^{2}$.

Ce dossier, pour l'essentiel, est centré sur les discours suscités par les attentats qui ont frappé la France en 2015 et 2016, certains auteurs les mettant toutefois en perspective dans le temps (Isabelle Garcin-Marrou et Isabelle Hare remontent aux attentats parisiens de 1995) ou dans l'espace (l'étude de Myriam Cremer et Rolf Kailuweit porte aussi sur l'attentat du 11 mars 2004 à Madrid). Les discours analysés relèvent en outre de sphères d'utilisation du langage (Bakhtine, 1984) distinctes : la presse écrite (version numérique comprise) dans les articles de Maëlle Bazin, d'I. Garcin-Marrou et I. Hare, et de Katharina Niemeyer, avec dans les trois cas une focalisation sur la presse nationale la plus « légitime» (Le Monde en particulier); les médias audiovisuels, et plus précisément les émissions télévisées improvisées pour couvrir les événements «en direct », pour Pierre Lefébure, Émilie Roche et Claire Sécail ; mais aussi les pouvoirs publics, et en particulier l'Élysée, pour Laurie Boussaguet et Florence Faucher; et enfin les mémoriaux populaires érigés dans la rue après les attentats pour M. Cremer et R. Kailuweit. Embrasser la diversité de cette production discursive était essentiel afin de pouvoir dépasser de simples constats localisés, et avancer ainsi dans une meilleure compréhension de ce qui se joue, de manière globale, au travers des discours post-attentats.

\section{Discours émotionnés, discours émouvants}

Le premier constat qui se dégage à cet égard de l'ensemble des contributions à ce dossier est l'omniprésence des émotions dans les discours postattentats : cela vaut pour les discours politiques, les prises de parole de

2. Ce n'est pas la première fois que Mots. Les langages du politique publie des articles sur le sujet. Dans un récent numéro, on a déjà pu lire une étude comparative des discours de l'exécutif politique en France et en Belgique consécutifs aux attentats de 2015 et 2016 (Wagener, 2018), ainsi qu'une analyse des usages du mot guerre dans deux quotidiens français (Le Monde et Le Figaro) après les attentats de janvier et novembre 2015 (Veniard, 2018). Par le passé, la revue s'était également penchée sur la sémiologie des pancartes dans les rassemblements en réaction à l'attentat contre la rédaction de Charlie Hebdo (Bazin, 2016), sur le traitement des attentats du 11-Septembre dans la presse française et italienne (Ramel, 2004; Cabasino, 2004), et sur les enjeux afférents à la nomination même de ces attentats (Fragnon, 2007; Calabrese Steimberg, 2008). 
citoyens ordinaires, et peut-être surtout pour les discours des médias que l'on peut qualifier de «dominants». Ce sont à la fois des discours "émotionnés» (Plantin, 2011) et émouvants, chargés d'émotion et, pour certains, visant à jouer sur les émotions des personnes auxquelles ils sont adressés.

À la suite de Gérôme Truc (2016, p. 17-54), la plupart des contributions portant sur le discours médiatique envisagent la sémiotisation3 des émotions postattentats en termes de cadrage (Goffman, 1991 [1974] ; Gamson, 1992 ; Entman, 1993). Ce que mettent en évidence les différents auteurs est une oscillation entre, d'un côté, la représentation de paroles, de témoignages «à forte charge émotionnelle» (P. Lefébure, É. Roche et C. Sécail), culminant dans la représentation d'un «peuple-émotion» (terme emprunté par M. Bazin à Pierre Rosanvallon), et, de l'autre, un effort de rationalisation du discours : «À peine est-on informé du drame et saisi par l'intensité émotionnelle que l'on est conjointement invité à, voire sommé de considérer froidement des éléments plus généraux ", relèvent ainsi $P$. Lefébure, É. Roche et $C$. Sécail. Cette mise à distance s'effectue généralement par la convocation de discours experts - ce qui n'empêche pas toutefois que cette parole experte puisse être elle-même "émotionnée »... La perspective historique proposée par I. Garcin-Marrou et I. Hare permet de constater en la matière une évolution patente du cadrage médiatique : contrairement à ce qui s'observe lors de la vague d'attentats du milieu des années 1990, la société française est représentée en 2015-2016 «comme une entité souffrant à l'unisson des victimes directes ", l'émotion populaire en venant à occuper une place centrale dans la couverture médiatique des attentats.

De ce point de vue, discours médiatiques et discours politiques semblent fonctionner comme des vases communicants. À la relecture des discours prononcés par le président de la République après les attentats de 2015 et 2016 (Boussaguet, Faucher), on est frappé par la place qu'occupent, là aussi, les émotions : les subjectivèmes (Kerbrat-Orecchioni, 1980) sont nombreux parmi les dénominations et désignations qualifiantes employées pour décrire les attentats. Et tant dans les discours - très contrôlés, réglés - du président François Hollande que dans les discours médiatiques, l'émotion apparaît tout à la fois « dite », «montrée » et "étayée» (Micheli, 2013), avec des dynamiques et des emboîtements entre ces différents modes de sémiotisation des émotions d'un discours à l'autre. Aux «larmes du pouvoir» (Le Bart, 2018) répond ainsi le «peuple en larmes » qu’instituent les médias, et réciproquement.

3. Nous reprenons ce terme à Raphaël Micheli pour qui il désigne le fait de «rendre manifeste [l'émotion] au moyen de signes ». Raisonner en ces termes présente l'intérêt de maintenir « une distinction de principe entre le registre du sémiotique et le registre de l'éprouvé. En effet, si l'on dit qu'un locuteur "sémiotise" une émotion, on indique uniquement qu'il rend une émotion manifeste au moyen de signes, sans présupposer qu'il éprouve effectivement cette émotion (même si cela peut bien entendu être le cas)» (Micheli, 2013). 
Si rien n'oblige à considérer qu'il y a derrière les émotions manifestées dans un discours des émotions réellement éprouvées par le locuteur (Micheli, 2013), la charge émotionnelle des discours médiatiques concernant les attentats étudiés ici semble néanmoins tenir en grande partie au rapport des journalistes à ces événements. Une proximité géographique, mais aussi professionnelle (dans le cas des attentats de Charlie Hebdo) et générationnelle (pour ceux du 13 novembre 2015) avec les victimes les conduit à basculer dans le registre de l'« information-émotion» (Marion, 1994) et à pratiquer un «journalisme d'empathie » (K. Niemeyer, l'expression vient des journalistes eux-mêmes) - ce qu'illustre particulièrement bien l'enquête menée par K. Niemeyer auprès de la rédaction du Monde. C'est aussi sous cet angle que peut se comprendre la place qu'occupent désormais (au moins depuis le 11-Septembre) dans les discours post-attentats des grands médias occidentaux les victimes, rescapés et témoins directs, et qui s'accompagne d'un accent mis, dans ces circonstances, sur les genres du portrait, du micro-trottoir et du reportage, mais aussi sur des formes d'hybridation entre productions médiatiques professionnelles et productions amateurs qui sont propices, comme le fait remarquer $M$. Bazin, à un effet de proximité vecteur d'émotions. Le discours médiatique favorise de la sorte la communion sociale, le rassemblement et le recueillement collectif, que vise également à produire, comme le montrent L. Boussaguet et F. Faucher, le discours politique.

Ainsi se constitue ce que l'on pourrait qualifier de discours politicomédiatique dominant, porteur d'«injonctions à se sentir concernés» (Truc, 2016) à forte valeur normative. L'étude des discours post-attentats de simples individus « ordinaires », tels qu'ils se donnent à voir dans les mémoriaux populaires de rue, en confirme la prégnance - qui peut donner à première vue face à ces mémoriaux l'impression d'un grand conformisme - en même temps qu'elle montre comment peuvent, par rapport à lui, émerger des distances critiques, des réserves, voire des contre-discours (Fraenkel, 2002 ; Sánchez-Carretero éd., 2011; Truc, 2016). C'est tout l'intérêt ici de la contribution de M. Cremer et R. Kailuweit, qui explorent les ressources linguistiques et rhétoriques mises en œuvre pour exprimer une émotion a priori indicible, parce qu'à la fois inconvenante (par rapport aux normes morales des sociétés occidentales) et contrevenante (par rapport au discours dominant), à savoir le désir de vengeance. La prise en compte de cet autre niveau - et genre - de discours impose dès lors de complexifier le tableau. Si l'on peut de prime abord penser, en se limitant au discours politico-médiatique dominant, qu'il n'y a rien d'autre à voir dans les discours post-attentats que la fabrication d'un consensus, l'étude attentive de ces discours dans leur pluralité conduit plutôt à interroger la portée de ce consensus, et ses limites. 


\section{Portée et limites du consensus post-attentats}

La tentation est forte de souligner le décalage entre d'un côté la violence des attentats perpétrés et de l'autre la fragilité des discours, ces derniers étant volontiers considérés comme des symboles de l'impuissance des démocraties face au terrorisme. Une telle asymétrie mérite pourtant réflexion. Comme le suggèrent les contributions présentées dans ce numéro, les attentats suscitent une production symbolique extrêmement riche et diverse qui, si elle ne suffit évidemment pas à effacer la violence (ni même à la faire cesser), remplit des fonctions anthropologiques fondamentales. Il est clair notamment que ces discours précipitent la formation d'une communauté émotionnelle en réaction à l'attaque et activent des sentiments de commune appartenance, à commencer par le sentiment national, sur lequel le discours politico-médiatique dominant met prioritairement l'accent. Tout nous enjoint dans ces circonstances à nous sentir concernés par l'attentat et à compatir au sort de nos concitoyens qui en sont victimes.

Pour des raisons qui tiennent à leur ancrage social et géographique, mais aussi à l'idée qu'ils se font de leur rôle, les journalistes eux-mêmes n'y échappent pas, de sorte qu'il n'est généralement pas besoin au pouvoir politique d'exercer sur eux une quelconque pression ou censure pourvoir leurs discours médiatiques converger vers un cadrage consensuel des événements ne tolérant guère d'exception (Delage, 2006). Ainsi les analyses des corpus médiatiques proposées dans ce dossier donnent-elles à voir une orchestration sans chef d'orchestre dans la mise en mots des attentats et dans la production des normes cadrant l'attitude qu'il convient d'adopter face à eux. Si la démarche comparative de certains auteurs permet de nuancer le tableau, ou plutôt de le situer, on demeure frappé par la prévisibilité des productions journalistiques, en particulier dans le traitement «à chaud» des attentats par les chaînes de télévision (P. Lefébure, É. Roche et C. Sécail), ou dans la publication des portraits de victimes dans la presse écrite (K. Niemeyer). Dans un cas comme dans l'autre, l'accent mis sur la dimension humaine du drame favorise des mécanismes d’identification aux personnes qui en ont été directement victimes en même temps qu'elle donne corps à la communauté émotionnelle, qui tend incidemment à se confondre avec la communauté nationale (Butler, 2005 [2004]).

Reste que la portée symbolique de la parole étatique joue en elle-même, dans la production du consensus post-attentat, un rôle fondamental (Boussaguet, Faucher, 2016). Il est frappant de constater que c'est au moment même où l'État se trouve défié dans sa prétention régalienne à «monopoliser la violence physique légitime » (selon la fameuse formule de Max Weber) qu’il tente de sauver la face et de retrouver de sa superbe en s'efforçant de «monopoliser la violence symbolique légitime» (Bourdieu, 2012, p. 14). L'État et ceux qui 
le représentent - en premier lieu le chef d’État (Guigo, 2016) - s’investissent avec une énergie toute particulière pour imposer un cadrage de l'événement, un registre explicatif, et rappeler en même temps le pouvoir agissant (et réagissant) de l'exécutif. À la stupeur suscitée par l'attentat terroriste, celui-ci répond par un raidissement symbolique : «il sait de quoi il s'agit et il sait ce qu'il doit faire », comme l'écrivent dans ce numéro L. Boussaguet et F. Faucher. Et là encore, les émotions occupent une place centrale. L'exécutif dit ressentir, et dit quoi ressentir : jouant de la performativité de la rhétorique émotionnelle, il impose une grammaire de la sensibilité particulièrement rigide (Le Bart, 2018), qui s'incarne aussi dans des cérémonies ou des gestes rituels, tels que les minutes de silence ou le pavoisement. Mais ce faisant, en disant explicitement qui doit ressentir quoi, et comment le montrer, l'État s'expose aussi à la parole critique des citoyens (Truc, 2016, p. 147-171).

À trop vouloir produire, ou mettre en scène, le consensus, le risque est donc paradoxalement d'en rendre les limites manifestes. Car, derrière l'unanimisme des discours émotionnés, les éléments de dissensus sont aussi là, repérables dès le départ pour qui prend le temps de regarder finement les choses, dans les mémoriaux de rue comme sur les plateaux télévisés. Ainsi P. Lefébure, É. Roche et $C$. Sécail font-ils remarquer que le soir même des attentats du 13 novembre des «brèches » dans le consensus attendu sont ouvertes à l'antenne par des experts, ou des représentants politiques. On sait en effet que si des attentats comme ceux qu'a connus la France en 2015 et 2016 créent des situations de crise propices à un esprit d' "union nationale», ces situations n’impliquent pas pour autant la disparition, ou le dépassement sine die, des clivages politiques traditionnels et des enjeux partisans (Baudot, 2015) - et ce d'autant moins que les attentats se répètent, et que cette répétition affaiblit la dynamique d'union, comme le soulignent L. Boussaguet et F. Faucher. Si la parole étatique peut donc, dans ces circonstances particulières, s’imposer aux autres plus que d'ordinaire, les désaccords et divergences de points de vue, constitutifs d'une société démocratique, demeurent. Dès lors, la question principale devient celle des conditions auxquelles ils peuvent être, en contexte post-attentats, formulés et, peut-être plus encore, entendus. Pour y répondre, il faudrait encore ajouter aux contributions rassemblées dans ce numéro d'autres études, portant sur d'autres genres de discours et d'autres sphères d'utilisation de langage que ceux explorés ici : les médias indépendants et alternatifs ${ }^{4}$, les forums internet et les réseaux socio-numériques 5 , ou

4. À propos des attentats du 11-Septembre, voir par exemple, dans des styles différents, Entman (2003) ou France et Motta (2017), et sur ceux du 11 mars 2004 à Madrid, Chueca (2012).

5. Ce point suscite déjà une littérature abondante. Pour une première approche, on pourra se référer prioritairement à Robinson (2005), Flesher Fominaya (2011), Badouard (2016) et, s'agissant du cas particulier de Twitter, à Giglietto et Lee (2015), Smyrnaios et Ratinaud (2017), et Eriksson (2018). 
encore les prises de parole d'intellectuels 6 . Il était impossible, dans le cadre de ce dossier, de prétendre à l'exhaustivité. Mais notre espoir est qu'il fasse naître de nouvelles recherches sur le sujet, plutôt que de donner le sentiment que nous en avons fait le tour.

\section{Références}

ARON Raymond, 1962, Paix et guerre entre les nations, Paris, Calmann-Lévy.

BAKHTINE Mikhaïl, 1984 [1952-1953], «Les genres du discours», dans Esthétique de la création verbale, Paris, Gallimard, p. 265-272.

BADOUARD Romain, 2016, "Je ne suis pas Charlie”. Pluralité des prises de parole sur le web et les réseaux sociaux», dans Le défi Charlie : les médias à l'épreuve des attentats, P. Lefébure et C. Sécail éd., Paris, Lemieux éditeur, p. 187-219.

BAudot Pierre-Yves, 2015, "Le 11-janvier : crise ou consensus?», La Vie des idées, http://www.laviedesidees.fr/Le-11-janvier-crise-ou-consensus.html (consulté le 14 juin 2018).

BAzıN Maëlle, 2016, «Brandir un crayon : geste-emblème des rassemblements postattentats de janvier 2015 ", Mots. Les langages du politique, no ${ }^{0} 110$, p. 67-81.

Bourdieu Pierre, 2012, Sur l'État. Cours au collège de France (1989-1992), Paris, Seuil.

BousSAgUet Laurie, FAUCHER Florence, 2016, « Mobiliser des symboles pour répondre au terrorisme : l'exécutif français face aux attentats de 2015 à Paris ", LIEPP Policy Brief, no 28, https://spire.sciencespo.fr/hdl:/2441/6ndhje5f5f8gtrlj1s95p1phgt/ resources/2016-faucher-mobiliser-des-symboles.pdf (consulté le 14 juin 2018).

BUTLER Judith, 2005 [2004], Vie précaire : les pouvoirs du deuil et de la violence après le 11 septembre 2001, J. Rosanvallon et J. Vidal trad., Paris, Éditions Amsterdam.

CABASINo Francesca, 2004, "Les attentats du 11 septembre : émotion et raison dans la presse en France et en Italie », Mots. Les langages du politique, nº 75, p. 21-33.

CALABRESE Steimberg Laura, 2008, «Les héméronymes : ces évènements qui font date, ces dates qui deviennent évènements », Mots. Les langages du politique, n०88, p. $115-128$.

Chueca Miguel, 2012, "Les attentats du 11 mars 2004 à Madrid et les "théories de la conspiration" ", Agone, n² 47, p. 105-143.

Cousin Bruno, VITALE Tommaso, 2014, «Le magistère intellectuel islamophobe d'Oriana Fallaci : origines et modalités du succès italien de la "Trilogie sur l'Islam et sur l'Occident” (2001-2006) », Sociologie, vol. V, nº1, p. 61-79.

DeLAGE Christian, 2006, "Une censure intériorisée? Les premières images des attentats du 11 septembre 2001 », Ethnologie française, vol.XXXVI, nº 1, p. 91-99.

DeLDicque Timothée, 2016, "Le rôle des “philosophes médiatiques". Construction symbolique des événements", dans Le défi Charlie : les médias à l'épreuve des attentats, P. Lefébure et C. Sécail éd., Paris, Lemieux éditeur, p. 315-345.

ENTMAN Robert, 2003, "Cascading activation: contesting the White House's frame after 9/11», Political Communication, vol.XX, n 4, p. 415-432.

- 1993, «Framing: toward clarification of a fractured paradigm », Journal of Communication, vol.XLIII, nº 4 , p. 51-58.

6. Voir par exemple Lagarde (2005), Cousin et Vitale (2014) et Deldicque (2016). 
ERIKSSON Moa, 2018, «Pizza, beer and kittens: negotiating cultural trauma discourses on Twitter in the wake of the 2017 Stockholm attack», New Media \& Society, OnlineFirst, http://journals.sagepub.com/doi/full/10.1177/1461444818765484 (consulté le 14 juin 2018).

FERRAGu Gilles, 2019 (à paraître), "L'écho des bombes : l'invention du terrorisme “à l'aveugle" (1893-1895)», Ethnologie française.

FLESHER FominAYA Cristina, 2011, «The Madrid bombings and popular protest: misinformation, counter-information, mobilisation and elections after '11- $M$ ' », Contemporary Social Science, vol. VI, nº 3 , p. 289-307.

FrAen Kel Béatrice, 2002, Les écrits de septembre: New York 2001, Paris, Textuel.

FRAGNON Julien, 2007, "Quand le 11-Septembre s'approprie le onze septembre : entre dérive métonymique et antonomase », Mots. Les langages du politique, n० 85 , p. 83-95.

France Pierre, MotTa Alessio, 2017, «En un combat douteux. Militantisme en ligne, “complotisme" et disqualification médiatique : le cas de l'association ReOpen911», Quaderni, no 94 , p. 13-27.

Gamson William A., 1992, Talking Politics, Cambridge, Cambridge University Press.

GIGlietto Fabio, LeE Yenn, 2015, «To be or not to be Charlie: Twitter hashtags as a discourse and counter-discourse in the aftermath of the 2015 Charlie Hebdo shooting in France ", dans Proceedings of the $5^{\text {th }}$ Workshop on Making Sense of Microposts colocated with the $24^{\text {th }}$ International World Wide Web Conference (WWW 2015), M. Rowe, M. Stankovic et A.-S. Dadzie éd., vol. 1395, p. 33-37.

GoffmAn Erving, 1991 [1974], Les cadres de l'expérience, I. Joseph trad., Paris, Minuit.

GUIGo Pierre-Emmanuel, 2016, «L'étoffe présidentielle à l'épreuve : la communication de F. Hollande pendant les attentats », dans Le défi Charlie : les médias à l'épreuve des attentats, P. Lefébure et C. Sécail éd., Paris, Lemieux éditeur, p.79-114.

KeRBRAT-ORECCHIONI Catherine, 1980, L'énonciation de la subjectivité dans le langage, Paris, Armand Colin.

LAGARDE François, 2005, "Penser l'impensable: le 11 Septembre des penseurs français ", French Politics, Culture \& Society, vol.XXIII, nº 2, p. 91-100.

LANÇON Philippe, 2018, Le lambeau, Paris, Gallimard.

LE BART Christian, 2018, Les émotions du pouvoir : larmes, rires, colères des politiques, Malakoff, Armand Colin.

LefÉBURE Pierre, SÉCAIL Claire éd., 2016, Le défi Charlie : les médias à l'épreuve des attentats, Paris, Lemieux éditeur.

MALANDAIn Gilles, 2012, "Les sens d'un mot : “attentat”, de l'Ancien Régime à nos jours», La Révolution française, nº 1 , https://journals.openedition.org//rf/368 (consulté le 14 juin 2018).

MARION Philippe, 1994, «L'affect télévisuel : les funérailles du roi Baudouin », Hermès, no 13-14, p. 315-332.

MICHELI Raphaël, 2013, «Esquisse d'une typologie des différents modes de sémiotisation verbale de l'émotion », Semen, n³ 35, http://journals.openedition.org/semen/9795 (consulté le 14 juin 2018).

PLANTIN Christian, 2011, Les bonnes raisons des émotions : principes et méthode pour l'étude du discours émotionné, Berne, Peter Lang.

RAMEL Frédéric, 2004, "Presse écrite et traitement immédiat du 11 septembre : un imaginaire occidental réactivé ? », Mots. Les langages du politique, nº 76, p. 113-126.

RımÉ Bernard, 2005, Le partage social des émotions, Paris, PUF. 
RoBINSON Laura, 2005, «Debating the events of September $11^{\text {th }}$ : discursive and interactional dynamics in three online fora », The Journal of Computer-Mediated Communication, vol.X, no 4 , https://onlinelibrary.wiley.com/doi/full/10.1111/j.1083-6101.2005. tb00267.x (consulté le 14 juin 2018).

SALAS Denis, 2018, La foule innocente, Paris, Desclée de Brouwer.

SAlomé Karine, 2010, L'ouragan homicide : l'attentat politique en France au xixe siècle, Seyssel, Champ Vallon.

SÁNCHEZ-CARRETERo Cristina éd., 2011, El Archivo del Duelo: análisis de la respuesta ciudadana ante los atentados del 11 de marzo en Madrid, Madrid, CSIC.

Sıмко Christina, 2015, The Politics of Consolation: Memory and the Meaning of September 11, Oxford, Oxford University Press.

Smyrnalos Nikos, Ratinaud Pierre, 2017, «The Charlie Hebdo attacks on Twitter: a comparative analysis of a political controversy in English and French », Social Media + Society, vol. III, $\mathrm{n}^{0} 1$, p. 1-13.

TRUC Gérôme, 2016, Sidérations : une sociologie des attentats, Paris, PUF.

VENIARD Marie, 2018, «La presse devant les attentats terroristes : usages journalistiques du mot guerre (Paris, 2015) », Mots. Les langages du politique, nº 116, p. 91-109.

WAGENER Albin, 2018, «Réactions post-attentats en France et en Belgique : étude comparative des discours de l'exécutif politique », Mots. Les langages du politique, no116, p. 111-130. 